\title{
Resilience Factors and Their Connection with Behavioural and Emotional Problems Among Disadvantaged Adolescents Involved in The Child Welfare System
}

\author{
Barnabás Oláh ${ }^{1}$, Beáta Kovács-Tóth ${ }^{2}$ \\ Department of Behavioural Sciences, University of Debrecen ${ }^{1,2}$
}

\begin{abstract}
Traumatization occurs more frequently among disadvantaged children involved in the child welfare system. Consequences may include emotional or behavioural problems, and difficulties in social relationships. The term of resilience refers to the successful adaptation despite risk and adversity. The focus of the study was to assess the resilience and its influencing factors in disadvantaged adolescents involved in the Hungarian child welfare system, as well as the relationship between resilience and behavioural and emotional problems. Our sample consisted of adolescents ( $N=154,75$ girls) aged 12 to 17 living in group homes. The following questionnaires were administered: The Child and Youth Resilience Measure (CYRM-28); the Strengths and Difficulties Questionnaire (SDQ); 5 question groups from the Health Behaviour in School-Aged Children questionnaire (HBSC-2014) and a self-designed structured interview for caregivers. $21 \%$ of the adolescents showed low resilience, $71 \%$ demonstrated moderate resilience, whereas $8 \%$ of them were highly resilient. Resilience showed a moderate negative correlation with behavioural and emotional problems. Automatic linear modelling revealed that prosocial behaviour, quality of maternal bond, peer support and physical activity together account for $38 \%$ of the variance. $21 \%$ of adolescents living in group home showed low resilience. The extent of resilience showed a negative connection with the number of behavioural and emotional problems. Promoting social support, peer relations and regular physical activity may affect resilience positively among children. Further research is needed in the topic to better understand the causal relations and explore additional factors of resilience.
\end{abstract}

Keywords: adaptation, childhood abuse, group home, protective factors, psychopathological symptoms 